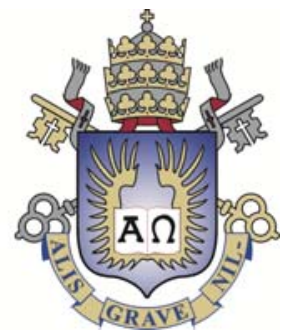

Moacyr Ferreira

Implicações estratégicas de empresas que competem globalmente por meio de alianças e redes:

O caso Nokia Smart Devices

Dissertação de Mestrado

Dissertação apresentada ao Programa de Pósgraduação em Administração de Empresas da PUCRio como requisito parcial à obtenção do título de Mestre em Administração de Empresas.

Orientadora: Prof ${ }^{a}$. Teresia Diana Lewe van Aduard de Macedo-Soares 


\title{
Moacyr Ferreira
}

\author{
Implicações Estratégicas de Empresas que \\ competem Globalmente por meio de Alianças \\ e Redes: O caso Nokia Smart Devices
}

Dissertação apresentada como requisito parcial para obtenção do grau de Mestre pelo Programa de PósGraduação em Administração de Empresas da PUC-Rio. Aprovada pela Comissão Examinadora abaixo assinada.

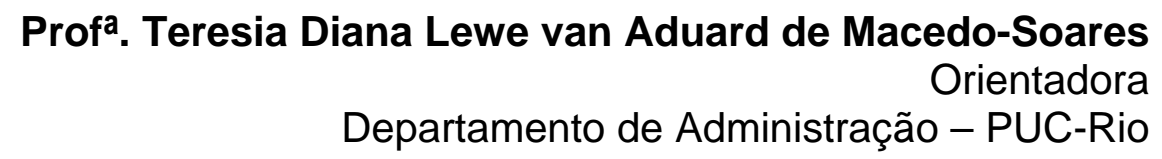

Profa. Maria Fatima Ludovico de Almeida Programa de Pós-Graduação em Metrologia - PUC-Rio

Prof ${ }^{a}$. Irene Raguenet Troccoli

UNESA

Profa. Mônica Herz

Vice-Decana de Pós-Graduação do CCS - PUC-Rio 
Todos os direitos reservados. É proibida a reprodução total ou parcial do trabalho sem autorização da Universidade, do autor e da orientadora.

\section{Moacyr Ferreira}

Graduou-se em Engenharia Elétrica pela Escola Politécnica da USP (POLI-USP) em 1989, e em Economia pela Faculdade de Administração, Economia e Ciências Contábeis da USP (FEA-USP), em 1994. Atua como profissional há mais de 20 anos, com passagens pelo setor elétrico e, atualmente, no setor de telecomunicações, exerce atividades de vendas na Nokia Solutions Networks, com experiência internacional na América Latina, nos Estados Unidos e na Europa.

Ficha Catalográfica

Ferreira, Moacyr

Implicações Estratégicas de Empresas que competem Globalmente por meio de Alianças e Redes: O caso Nokia Smart Devices/ Moacyr Ferreira; orientadora: Teresia Diana Lewe van Aduard de Macedo-Soares - 2014.

257f.: il. (color.); $30 \mathrm{~cm}$

Dissertação (mestrado) - Pontifícia Universidade Católica do Rio de Janeiro, Departamento de Administração, 2014.

Inclui bibliografia e anexos

1. Alianças Estratégicas. 2. Estratégia global. 3. Telecomunicações móveis. I. Macedo-Soares, Teresia Diana Lewe van Aduard de. II. Pontifícia Universidade Católica do Rio de Janeiro. Departamento de Administração. III. Título. 
Para minha eterna "menininha":

RaphaelleValentine. 


\section{Agradecimentos}

À minha orientadora, Prof ${ }^{\mathrm{a}}$ T. Diana L. v. A de Macedo-Soares, fonte de aprendizado e inspiração, que me mostrou que, para ser um Mestre, é preciso mais do que conhecimento. É preciso ter o interesse legítimo em orientar outras pessoas no caminho do saber, o que ela demonstrou com paciência e perseverança.

Aos Professores Maria Fátima Ludovico de Almeida, Irene Raguenet Troccoli e Marcos Cohen, pelo interesse e respeito, pela concordância em participar da Comissão Examinadora, apesar das várias outras tarefas nas quais estão envolvidos, e pela contribuição por meio de comentários que enriqueceram esta pesquisa.

Aos Professores do Mestrado do IAG-PUC, sem exceção, com os quais tive o prazer de entrar em contato nas disciplinas por eles ministradas, pela contribuição de cada um deles para o meu processo de amadurecimento acadêmico, enriquecendo os meus conhecimentos por meio de aulas, textos e comentários trazidos ao longo do curso.

Aos amigos que aceitaram o desafio de colaborar com a participação na resposta aos questionários e entrevistas, e, assim, viabilizando a conclusão deste trabalho, apesar das adversidades enfrentadas no processo de coleta de dados.

Especialmente à família. Adriana, minha esposa, e Raphaelle Valentinne, minha filha. Agradecimento por estarem sempre próximas, e, ainda que eu lhes tenha "roubado" vários minutos da convivência, pelo apoio que foi indispensável à concretização de um objetivo especial. 


\section{Resumo}

Ferreira, Moacyr; Macedo-Soares, Teresia Diana Lewe van Aduard de. Implicações estratégicas de empresas que competem globalmente por meio de alianças e redes: O caso Nokia Smart Devices; Rio de Janeiro, 2014. 257p. Dissertação de Mestrado - Departamento de Administração, Pontifícia Universidade Católica do Rio de Janeiro.

O setor de telecomunicações móveis figura entre os mais dinâmicos e complexos ambientes de negócios. Indústrias de TIC (Tecnologia de Informação e Comunicações), antes separadas, começam a mesclar-se, pouco a pouco, dificultando a delimitação de fronteiras entre os participantes que, de competidores, em muitas situações, passam à condição de parceiros, com vistas à complementação de recursos e de competências. Esta pesquisa tem como objetivo a verificação da adequação da estratégia da Nokia Smart Devices à indústria de telefonia móvel, considerando suas alianças e a estratégia competitiva global. A investigação foi conduzida com o uso da metodologia de estudo de caso. Múltiplas fontes de evidência foram utilizadas para permitirem a convergência triangular dos dados. A interpretação e a análise dos dados foram realizadas com auxílio do referencial teórico identificado, e baseadas no Global Strategic Network Analysis - SNA - framework, de Macedo-Soares (2011). Os resultados da pesquisa apontam a presença de ameaças que desafiam a liderança de empresas, muitas vezes tidas como sólidas. O entendimento das implicações estratégicas dos fatores macroambientais (estruturais e relacionais) e dos fatores organizacionais é fundamental para a composição de uma estratégia consistente, capaz de alavancar as forças criadas pela organização ou pela rede, ou de minimizar as fraquezas, com o propósito de aproveitar as oportunidades oferecidas pelo ambiente, e, ainda, de mitigar as ameaças, na busca pela geração de vantagem competitiva.

\section{Palavras-chave}

Alianças estratégicas; estratégia global; telecomunicações móveis; Nokia. 


\section{Abstract}

Ferreira, Moacyr; Macedo-Soares, Teresia Diana Lewe van Aduard de. Strategic Implications of Alliances \& Networks of Companies Competing at a Global Level: The case of Nokia Smart Devices. Rio de Janeiro, 2014. 257p. MSc. Dissertation - Departamento de Administração, Pontifícia Universidade Católica do Rio de Janeiro.

The mobile phone sector is among the most dynamic and complex business environment. ICT (Information and Communication Technology) industries, which were, to date, treated in a separated way, gradually start to converge, so that the boundaries among the participants thereof become hardly identifiable. In some cases, the members undertake the role of competitors and, in other occasions, they act as strategic partners, aiming at the complementation of resources and competencies. The present research intends to verify the adequacy of Nokia Smart Devices strategy to the Mobile Phone industry, considering its alliances and the global competitive strategy. The work adopted the case study methodology. For the collection of the required data, multiple sources and means to were used in accordance with the strategy of data and method triangulation, so as to ensure results' consistency. Data interpretation and analysis were carried out in the light of the research's theoretical references and pursuant to the Global Strategic Network Analysis - SNA framework, by Macedo-Soares (2011). The results point out the presence of threats that challenge companies' leadership which may sometimes be considered as very solid organizations. The correct comprehension of strategic implications of macroenvironmental (structural and relational) and organizational factors is fundamental to a correct and consistent strategy composition, that may leverage the strengths generated by the organization and its network, or even minimize the weaknesses, in order to benefit from opportunities offered by the sector while and to mitigate the threats there from, while reaching a competitive edge.

\section{Key- words}

Strategic alliances; global strategy; mobile telecommunication; Nokia. 


\section{Sumário}

$\begin{array}{ll}\text { Prefácio } & 16\end{array}$

1. Introdução 18

1.1. O Problema 18

1.2. Objetivos 23

1.2.1. Objetivo da Pesquisa Maior 23

1.2.2. Objetivos Final e Específico 24

1.2.3. Pressupostos 25

1.3. Relevância do Estudo 26

1.4. Delimitação do Estudo 26

1.5. Estrutura da Dissertação 28

2. Referencial Teórico 29

2.1. Revisão da Literatura $\quad 29$

2.1.1. A Telefonia Móvel e seu Ambiente Competitivo 30

2.1.2. Estratégia e Criação de Vantagem Competitiva 37

2.1.3. A Importância do Fator Inovação e sua Implicação
Estratégica

2.1.4. O Fator Liderança e sua Implicação Estratégica 84

2.2. Posicionamento teórico $\quad 87$

2.2.1. Tipologias e Conceitos Principais Adotados 87

2.2.2. Ferramental para Análise das Implicações Estratégicas 94

2.2.3. Variações, Construtos e Indicadores 96

2.2.3.1. Metodologia de Análise Estratégica - Global SNA
Framework

2.2.3.2. Listas de Referência - Global SNA Framework 98

2.2.3.3. Modelo - Global SNA 115

3. Metodologia de Pesquisa 117

3.1. Tipo e Método de Pesquisa 117

3.2. Unidade de Análise $\quad 121$

3.3. Seleção de Sujeitos $\quad 122$

3.4. Pesquisa Bibliográfica 126

3.5. Levantamento de Evidências 128

3.5.1. Elaboração do Questionário 128

3.5.2. Elaboração do Questionário 129

3.5.3. Levantamento Complementar 131

3.6. Tratamento das Evidências 131

3.7. Limitações Metodológicas 131 
4. Resultados 133

4.1. O Mercado de Telecomunicações 134

4.2. Nokia: Uma Perspectiva Histórica 140

4.3. Caracterização das Estratégias Competitivas da Nokia Smart Devices

4.4. Implicações Estratégicas dos Fatores Estruturais

(Macroambientais relevantes e dos principais atores globais) 150

4.5. As Implicações Estratégicas dos Fatores Organizacionais 158

4.6. Implicações Estratégicas das Alianças da Nokia Smart

Devices

170

4.7. A Ego-rede da Nokia Smart Devices 174

4.8. As Implicações Estratégicas dos Fatores Relacionais 175

4.9. O Desempenho da Nokia Smart Devices 179

5. Discussão dos Resultados 181

6. Conclusões 202

7. Referências Bibliográficas 209

Anexo I 219

Anexo II 237

$\begin{array}{ll}\text { Anexo III } & 257\end{array}$ 


\section{Lista de figuras}

Figura 1 - Estatísticas mundiais de telefonia móvel: período entre 2001 e 2013

Figura 2 - Market Share de fornecedores globais em telefonia móvel: período entre 2009 e 2012

Figura 3 - Valor da marca Nokia: período entre 2001 e 2012

Figura 4 - Orientação estratégica para a Nokia Smart Devices 47

Figura 5 - Modelo de mediação, segundo Liao, Kickul e Ma (2009) 69

Figura 6 - Modelo de inovação, ambiente e desempenho 76

Figura 7 - Efeito da incerteza e complexidade sobre o

gerenciamento da inovação

Figura 8 - Modelo de integração de práticas de gerenciamento de inovação, adaptação do alinhamento estratégico e resultado dos negócios

Figura 9 - Modelo para o sistema de indicadores e práticas de Inovação, segundo Ziviani, 2012

Figura 10 - Modelo Global SNA

116

Figura 11 - Ego Rede da Nokia Smart Devices

174 


\section{Lista de quadros}

Quadro 1 - Escolas de pensamento estratégico 38

Quadro 2 - Caracterização de estratégias, segundo a tipologia de Mintzberg (1998)

Quadro 3 - Conteúdos relacionados à caracterização da

estratégica, segundo Fahey e Randall (1998)

Quadro 4 - Características de orientações estratégicas

segundo Isoherranen e Kess, 2011a

Quadro 5 - Orientações mercadológicas segundo Isoherranen e Kess, 2011b

Quadro 6 - Classificação de estratégias segundo Isoherranen e Kess, 2011b

Quadro 7 - Orientação estratégica e posicionamento da Nokia

Smart Devices entre 1990 e 2009

Quadro 8 - Conceitos sobre exploration e exploitation

Quadro 9 - Estratégias de exploitation e exploration, segundo

Duysters e Dittrich, 2007

Quadro 10 - Dimensões de Inovação

66

Quadro 11 - Enfoques sobre inovação

70

Quadro 12 - Posicionamento dos autores sobre os conceitos de exploration e exploitation

Quadro 13 - Indicadores de conhecimento pertinentes à inovação

Quadro 14 - Indicadores de aprendizagem pertinentes à inovação

Quadro 15 - Indicadores de estratégia pertinentes à inovação

Quadro 16 - Indicadores de processos em inovação

81

Quadro 17 - Indicadores de resultados pertinentes à inovação

82

83

Quadro 18 - Tipologia de liderança, segundo Pearce et al. (2003)

Quadro 19 - Caracterização de estratégia de empresas

Multinacionais

88

Quadro 20 - Conteúdos relacionados à caracterização da

estratégia

Quadro 21 - Classificação das ligações estratégicas adaptada

à indústria de telecomunicações móveis

Quadro 22 - Construtos sobre Liderança

Quadro 23 - Construtos sobre Inovação

Quadro 24 - Modelo SNA: Categorias dos construtos/ descrição

Quadro 25 - Atributos necessários/ desejáveis para cada variável

principal independente para a gestão estratégica. Modelo GI

baseado em Macedo-Soares (2000)

Quadro 26 - Lista de referências dos Construtos relacionais para análise no âmbito da empresa, adaptados ao setor de telecomunicações móveis

Quadro 27 - Lista de referências dos Construtos relacionais para análise no âmbito da indústria, adaptados ao setor de 
Quadro 28 - Indicadores de desempenho para a avaliação de empresas fornecedoras de dispositivos de comunicação para o setor de telefonia móvel

Quadro 29 - Fatores Macroambientais

Quadro 30 - Atores pertencentes à Rede de Valor da Nokia

Smart Devices - Clientes

Quadro 31 - Atores pertencentes à Rede de Valor da Nokia

Smart Devices - Fornecedores

Quadro 32 - Atores pertencentes à Rede de Valor da Nokia

Smart Devices - Concorrentes

Quadro 33 - Atores pertencentes à Rede de Valor da Nokia

Smart Devices - Novos Entrantes

Quadro 34 - Atores pertencentes à Rede de Valor da Nokia Smart Devices - Substitutos

Quadro 35 - Atores pertencentes à Rede de Valor da Nokia

Smart Devices - Complementors

Quadro 36 - Percepções sobre os fatores organizacionais, captadas por meio do questionário

Quadro 37 - Implicações estratégicas dos fatores organizacionais da Nokia Smart Devices

Quadro 38 - Fatores relacionais para o âmbito da indústria Quadro 39 - Fatores relacionais para o âmbito da empresa 


\section{Lista de gráficos}

Gráfico 1 - Características do gerenciamento de portfólio de Alianças

Gráfico 2 - Distribuição dos participantes por área de atuação

Gráfico 3 - Distribuição dos participantes por nível hierárquico de atuação

Gráfico 4 - Distribuição dos participantes por tempo de atuação no setor de telefonia móvel

Gráfico 5 - Distribuição dos respondentes no tocante à tipologia de Mintzberg, 1998

Gráfico 6 - Distribuição dos respondentes no tocante à tipologia de Harzing, 2000

Gráfico 7 - Distribuição das orientações estratégicas segundo Isoherranen e Kess, 2011

Gráfico 8 - Distribuição da classificação estratégica segundo Miles et al., 1978

Gráfico 9 - Escopo geográfico da estratégia, segundo Fahey e Randall, 1998

Gráfico 10 - Escopo dos stakeholders da estratégia, segundo

Fahey e Randall, 1998

Gráfico 11 - Natureza das relações entre a NSD e seus parceiros Estratégicos 


\section{Lista de tabelas}

Tabela 1 - Número estimado de assinantes de telefonia móvel

134

Tabela 2 - Fabricantes de dispositivos de comunicação móvel

Tabela 3 - Distribuição de Assinantes de telefonia móvel por país

Tabela 4-Market Share de sistemas operacionais

Tabela 5 - Comportamento do usuário de telefonia móvel

Tabela 6 - Market Share de smartphones

Tabela 7 - Percepção dos respondentes sobre as alianças estratégicas da Nokia Smart Devices

Tabela 8 - Fatores importantes para o estabelecimento de alianças estratégicas

Tabela 9 - Fatores importantes para o estabelecimento de alianças estratégicas da Nokia Smart Devices 


\section{Lista de siglas}

TIC - Tecnologia de Informação e de Comunicação

GI - Generic Integrative

GSM - Global System for Mobile Communications

MNC - Multinational Companies

NSD - Nokia Smart Devices

R\&D - Research \& Development

SNA - Strategic Network Analysis 


\section{Prefácio}

O prefácio busca esclarecer a situação enfrentada pela Nokia Smart Devices durante a elaboração da pesquisa e suas consequências, particularmente no que se refere ao momento da conclusão do trabalho, que coincidiu com a tomada de decisão sobre a venda da empresa, prevista para ser concretizada até o primeiro trimestre de 2014.

O estudo das implicações dos fatores estrategicamente significativos estruturais, relacionais e organizacionais - é de grande importância neste mundo competitivo no qual se acham inseridas as organizações. Em particular, consideramos as indústrias de tecnologia de informação e de comunicação (TIC) e, mais especificamente, o setor de telecomunicações móveis, dada sua intensa dinâmica e alta complexidade.

A Nokia Smart Devices, atuante no setor de telefonia móvel, a exemplo de outras empresas envolvidas neste tipo de ambiente complexo e dinâmico, também foi impactada diretamente, ao longo do tempo, por estes fatores estratégicos.

Consideramos importante esclarecer alguns fatos recentes relacionados à Nokia Smart Devices, para uma melhor contextualização do momento enfrentado pela empresa durante o desenvolvimento e conclusão deste trabalho, e para a compreensão do impacto destes eventos sobre o mesmo.

O trabalho teve início em março de 2012, quando foi adotada como objeto de análise a divisão de negócios Nokia Smart Devices, por tratar-se de uma empresa que atua em âmbito global por meio de alianças e redes de ligações estratégicas, campo de pesquisa de grande relevância.

Ao longo da elaboração do trabalho, foram coletadas diversas evidências sobre o desempenho da empresa, desde o início da década de 2000 até o quarto trimestre de 2013. Em setembro de 2013, porém, foi tomada uma decisão de fundamental importância para o futuro da Nokia Smart Devices: a de liberação de venda da empresa para a Microsoft Corporation.

Muitos dos dados colhidos já apontavam para a dificuldade enfrentada pela empresa no mercado, devido à forte concorrência e à transformação tecnológica. 
Esta indústria, antes baseada em comunicação de voz, também passou a girarem torno da comunicação de dados, o que aumentou seu grau de complexidade.

Cerca de quase três anos após o estabelecimento da parceria estratégica entre as empresas Microsoft Corporation e Nokia Corporation, iniciada no início de 2010, a Nokia Corporation, em setembro de 2013, anunciou um acordo para a venda da Nokia Devices and Services para a Microsoft Corporation, por um valor total de €5,44 bilhões. O acordo incluía, além dos ativos físicos relacionados aos dispositivos de comunicação móvel, todas as licenças da Nokia (Devices and Services), por um período de 10 anos a partir da implantação do acordo, previsto para entrar em vigor no primeiro trimestre de 2014.

A concretização da venda da Nokia Smart Devices para a Microsoft em nada interfere na relevância deste estudo, pois a divisão Smart Devices permanecerá no mercado com a marca Nokia, pelo menos, até o primeiro trimestre de 2014 e, muito provavelmente, além deste período, já que a Microsoft pretende lançar mão da força da marca Nokia como fator de impulso para seu produto Windows aplicado aos dispositivos de comunicação móvel.

Devido ao momento delicado então atravessado pela empresa, em decorrência da venda, o departamento jurídico da Nokia do Brasil, adotou formalmente uma posição contrária à coleta de dados por meio de questionário estruturado aplicado aos seus empregados.

Assim, o aludido questionário, que seria submetido aos funcionários da Nokia do Brasil, foi substituído por um questionário apresentado aos profissionais da área de telecomunicações móveis no âmbito global.

A opção pela oitiva dos profissionais que não eram funcionários da Nokia Smart Devices foi considerada positiva, pois permitiu a obtenção de uma visão externa mais crítica sobre a empresa e seu desempenho. As opiniões foram expressas, a partir do conhecimento e experiência destes profissionais sobre a empresa, tanto por meio de questionários como de entrevistas semiestruturadas.

Apesar desta limitação, verificada em pleno desenrolar da pesquisa, os resultados apresentaram-se consistentes, levando-se em conta o cruzamento das evidências obtidas por meio das respostas aos questionários, das entrevistas e da documentação disponível ao público sobre a empresa. 